\title{
Measurement of Relative Void Surface Energies in Irradiated Metals by Small-Angle Scattering*
}

\author{
By R. W. HeNDRICKS AND J. SCHELTEN \\ Metals and Ceramics Division, Oak Ridge National Laboratory, Oak Ridge, Tennessee 37830, U.S.A.
}

(Received 29 April 1974)

\begin{abstract}
Voids formed in neutron irradiated metals are faceted on various crystallographic planes. This faceting has been shown to cause significant anisotropy in the small-angle scattering pattern [Epperson, J.E., Hendricks, R. W. \& Farrell, K. Studies of Voids in Neutron Irradiated Al Single Crystals. I. Small-Angle $X$-Ray Scattering and Transmission Electron Microscopy, To be published; Hendricks, R. W., Schelten, J. \& Schmatz, W. Studies of Voids in Neutron Irradiated Al Single Crystals. II. Small-Angle Neutron Scattering, To be published]. In the case of neutron-irradiated Al single crystals, the voids may be generally characterized as octahedral having (111) faces with a varying degree of truncation on (100) faces. If it is assumed that the voids are in an equilibrium shape (which can be achieved by a low-temperature anneal), the specific surface energies $\gamma_{h k l}$ and the truncation parameter $t$ are related by $\gamma_{100}=$ $\sqrt{ } 3(1-t) y_{111}$. Hendricks, Schelten \& Schmatz proposed that the truncation parameter $t$ could be measured by studying the anisotropy of the tail of the small-angle scattering curve. In such an experiment, the scattering is measured at constant $|\kappa|$ in a given plane in reciprocal space as a function of rotation of the crystal about an axis perpendicular to the diffraction plane. In this paper, the sensitivity of the proposed experiment to (i) the distribution of void sizes $N(D)$ and (ii) various crystallographic planes is numerically explored. It has been found that for certain low-index planes, the effect is sufficiently independent of $N(D)$, that reasonable estimates of $t$ can indeed be found. Thus, it is possible to obtain the specific void surface energies from small-angle diffuse scattering data.
\end{abstract}

* Research sponsored by the U. S. Atomic Energy Commission under contract with the Union Carbide Corporation.

J. Appl. Cryst. (1975), 8, 193

Small-Angle Scattering in Neutron Irradiated Copper

\author{
By C. Hofmeyr, K. IsEBeck AND R.M. MAYER \\ Atomic Energy Board, Pelindaba, Physical Metallurgy Division, P.B. X256, Pretoria, South Africa
}

(Received 15 July 1974)

\begin{abstract}
Work which has been started at Pelindaba on small-angle scattering in neutron irradiated copper is described. The facility consists of a $15 \mathrm{~m}$ long $S$-curved waveguide, which transmits a neutron spectrum with a maximum at 9A and a FWHM of 4A [Hofmeyr, C. \& Isebeck, K. (1964). Nucl. Instrum. Meth. 117, 9-16]. The collimator has an angular divergence of $0.01 \mathrm{rad}$ and a comparative measurement is made of a sample against a reference sample. Samples of ASARCO copper purified by annealing at $960 \mathrm{C}$ for 1 hour in a vacuum of $5 \times 10^{-4}$ torr were subsequently irradiated in the SAFARI reactor to $10^{19}$ and $10^{20} \mathrm{n} / \mathrm{cm}^{2}(E>0 \cdot 1 \mathrm{MeV})$ at estimated temperatures of $100 \mathrm{C}$ and $250 \mathrm{C}$ respectively. The smallangle scattering effect was shown to be due to radiation-induced damage through measuring a sample before irradiation, after irradiation and after annealing at $450 \mathrm{C}$ for 10 minutes, which restored the original scattering characteristics. The differential coherent-scattering cross sections (d $\sigma / \mathrm{d} \Omega$ ) have been plotted on a log-log scale against the wave vector K (Figure). Samples irradiated to $10^{20} \mathrm{n} / \mathrm{cm}^{2} \mathrm{show}$ an increase in scattering over the lower-dose-irradiated samples, the increased scattering being particularly strong for $K<0.05 \AA^{-1}$. For the former, the plot is linear over three orders of magnitude and has a gradient of $-4 \cdot 0$. Such a dependence is expected if voids are present (Porod's equation) provided that they are randomly oriented. In a polycrystalline material this will be true even if the voids are faceted. We therefore conclude that voids have been formed at this fluence and temperature, which accords with
\end{abstract}


data taken by Adda, Y. [Radiation-Induced Voids in Metals. USAEC Symposium series 26, p. 40. Albany: USAEC]. For the $10^{19} \mathrm{n} / \mathrm{cm}^{2}$ samples, a gradient of 3.6 was obtained indicating that a mixture of voids and loops contributes to the small-angle scattering (dislocation loops would produce a $K^{-3}$ dependence). The size and concentration of the voids have been estimated from that portion of the Porod plot, which is linear whilst another estimate of the voids as well as the loops has been made from the low- $K$ side of the Guinier plot (Table). In the sample irradiated to $10^{19} \mathrm{n} / \mathrm{cm}^{2}$, both loops and voids are present and the concentrations in the table were derived assuming only one or the other species to be present. For the high-temperature, high-dose sample, a definite interpretation can be made in terms of voids of density $\sim 10^{14} \mathrm{~cm}^{-3}$ and diameter between $130 \AA$ and $180 \AA$.

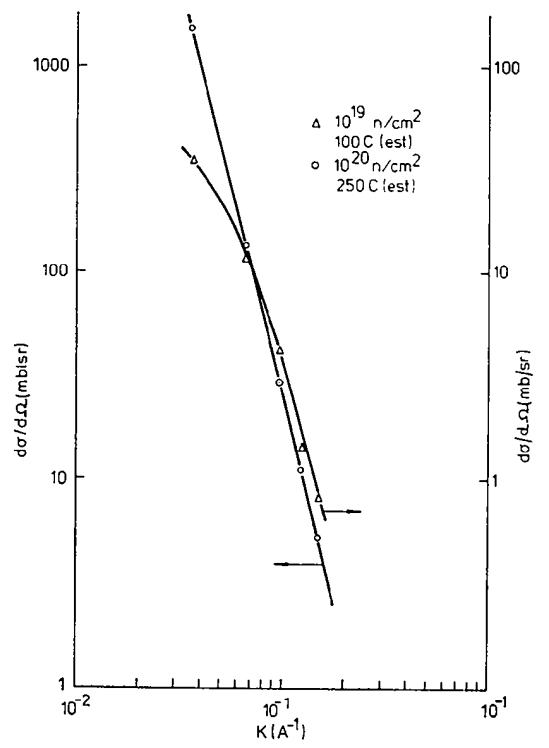

\begin{tabular}{ccccc} 
& \multicolumn{2}{c}{ Table } \\
Irradiation & Type & $\begin{array}{c}\text { Diameter } \\
(\AA)\end{array}$ & $\begin{array}{c}\text { Density } \\
\mathrm{cm}^{-3}\end{array}$ & Plot \\
$10^{19} \mathrm{n} / \mathrm{cm}^{2}$ & Loop & 85 & $4 \times 10^{16}$ & Guinier \\
$T=100 \mathrm{C}$ (est) & Void & 85 & $1 \times 10^{13}$ & Guinier \\
& & 65 & $7 \times 10^{13}$ & Porod \\
$10^{20} \mathrm{n} / \mathrm{cm}^{2}$ & Void & 130 & $6 \times 10^{14}$ & Guinier \\
$T \simeq 250 \mathrm{C}$ (est.) & & 180 & $8 \times 10^{13}$ & Porod
\end{tabular}

Figure

J. Appl. Cryst. (1975). 8, 194

\title{
The Determination of the Dislocation Structure in Copper Single Crystals from X-ray Diffraction Profiles
}

\author{
BY A. FREUND \\ Institut Max von Laue-Paul Langevin, B. P. 156 F-38042 Grenoble Cedex, France
}

(Received 29 April 1974)

\begin{abstract}
A careful analysis of X-ray diffraction profiles obtained from copper single crystals with dislocation densities between $10^{4}$ and $2 \times 10^{6}$ dislocations per $\mathrm{cm}^{2}$ has been performed. Lattice tilts and strains introduced by the as-grown dislocations have been deduced from double-crystal rocking curves recorded in a large range of Bragg angles, up to $89^{\circ}$. A new numerical deconvolution method [Mendes, M. \& Delestre, C. Submitted to Acta Cryst.] has been applied successfully to unfold the broadened diffraction patterns. The dislocation density $\varrho$ has been determined by etch-pit counting and by the application of Wilkens' theory [Wilkens, M. (1970). Phys, stat. sol. (a) 2, 359-370] to the diffraction profiles. Very good agreement has been obtained between the methods. The samples were free from low-angle grain boundaries. However, as a function of $\varrho$ the lattice tilts increased much faster than the strains, thus revealing the non-statistical distribution of the dislocations. The results indicate an arrangement of the dislocations in walls or cells as proposed by Bassim, M. N. \& Kuhlmann-Wilsdorf, D. [Cryst. Lattice Defects (1973). 4, 9-27]. The refined X-ray diffraction technique applied in this work has proved to be useful for obtaining information about the average arrangement of a great number of dislocations which is difficult to extract from topography or electron microscopy experiments. It can be used even for nearly perfect crystals.
\end{abstract}

